\title{
Review: evidence from 2 low quality screening studies does not show a reduction in death from prostate cancer
}

Ilic D, O'Connor D, Green S, et al. Screening for prostate cancer. Cochrane Database Syst Rev 2006;(3):CD004720.

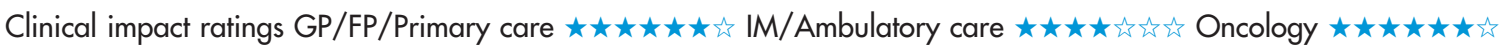

Does screening for prostate cancer reduce mortality?

\section{METHODS}

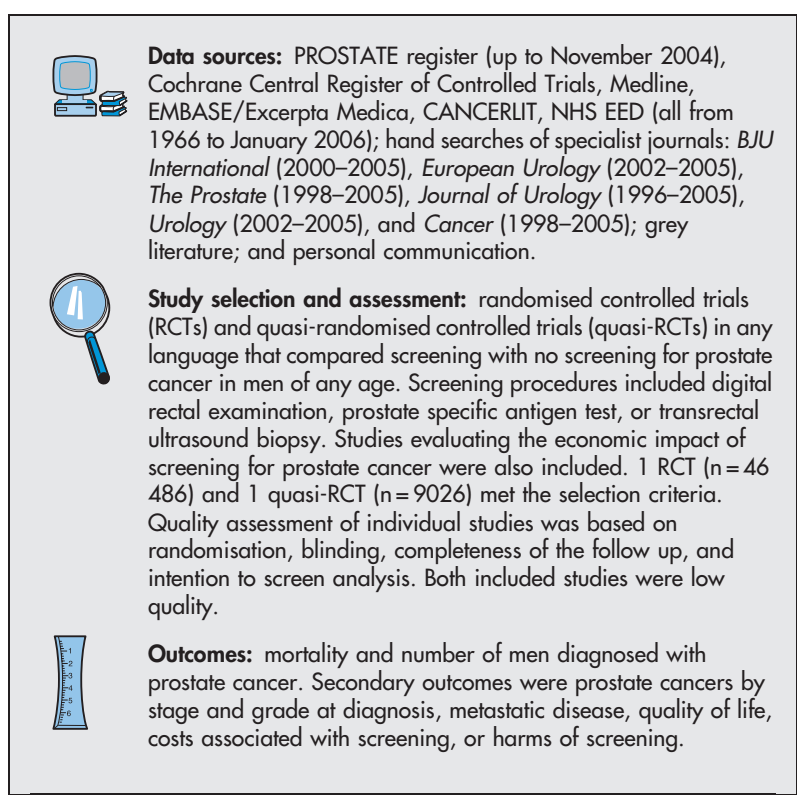

\section{MAIN RESULTS}

Reanalysis using intention to screen showed that groups did not differ for death from prostate cancer in I RCT and I quasi-RCT, respectively (table). More patients were diagnosed with prostate cancer in the screened group than in the unscreened group in the quasi-RCT at 15 years $(5.7 \% v 3.9 \%)$. In the RCT, $70 \%$ of cancer patients were diagnosed with stage B cancer at the first screening, increasing to $86 \%$ at follow-up. In the quasi-RCT, localised cancer was reported in $27 \%$ of cancer patients in the no screening group and in $84 \%$ in the screening group. Neither study assessed quality of life, costs associated with screening, or harms of screening.

For correspondence: $\mathrm{Mr} \mathrm{D}$ ilic, Monash University, Clayton, Victoria, Australia.dragan.ilic@med.monash.edu.au

Source of funding: no external funding

\section{CONCLUSION}

Evidence from 2 low quality screening studies does not show a reduction in death from prostate cancer.

\section{Commentary}

$\mathrm{T}$ he systematic review by lic et al concludes that randomised trials have yet to document that early detection of prostate cancer with prostate specific antigen (PSA) testing does more good than harm. Yet absence of proof is not proof of absence. Many feel the current $4 \%$ annual decline in prostate cancer mortality rates in the US must be attributable to PSA testing. However, evidence that screening explains the declines in mortality has been elusive. Perhaps improvements in treatment, including more widespread use of androgen deprivation for advanced cancer, explain some of this trend. Better trials would be welcome.

The US Prostate, Lung, Colorectal \& Ovary (PLCO) trial is an effectiveness trial of mass screening about 38000 randomised older men with annual PSA testing for 5 years. Men with elevated concentrations of PSA are referred to their own physicians. Controls get usual care, resulting in some degree of contamination in the control group. Screening will finish in 2007, with up to 10 years of additional follow up. The European Randomised study of Screening for Prostate Cancer (ERSPC) is an efficacy trial in 8 countries with about 200000 subjects. Although screening protocols vary, biopsy thresholds are standardised within countries, and evaluations for suspicious findings are pursued as part of the trial. Results are expected between 2007 and 2010. The designs of these 2 trials are complementary, and collaborative analyses are planned. The unique Prostate Testing for Cancer and Treatment (ProtecT) trial in the UK has a treatment trial nested within a screening trial. Men randomised to an offer of PSA screening also receive information on the treatment trial (surgery $v$ radiation $v$ active surveillance) should cancer be discovered. Recruitment should finish in 2008, with 10-15 years of additional follow up. Taken together, these trials should eventually determine whether early detection of prostate cancer does more good than harm.

In the meantime, some guidelines recommend counselling patients regarding the pros and cons of PSA testing and individualising the screening decision. Men should know that if they choose PSA screening, they substantially increase their lifetime risk of dealing with prostate cancer in exchange for an uncertain reduction in their risk of eventually dying from the disease.

Michael J Barry, MD Harvard Medical School Boston, Massachusetts, USA

Screening $v$ no screening for prostate cancer in men*

\begin{tabular}{lllll}
\hline Outcomes & Number of trials $(\mathbf{n})$ & Screening & No screening & RRI (95\% CI) \\
\hline Mortality at 8 years & 1 RCT $(46486)$ & $0.5 \%$ & $0.5 \%$ & $1 \%(-24$ to 33$)$ \\
Mortality at 15 years & 1 quasi-RCT $(9026)$ & $1.3 \%$ & $1.3 \%$ & $4 \%(-36$ to 68) \\
\hline
\end{tabular}

${ }^{*} \mathrm{RCT}=$ randomised controlled trial. Other abbreviations defined in glossary; RRI, NNT, and Cl calculated from data in article. 